Brit. J. industr. Med., 1952, 9, 65.

\title{
PENETRATION OF DUST PARTICLES AND SITES OF DUST STORES IN PNEUMOCONIOSIS
}

\author{
BY \\ GIACOMO MOTTURA \\ From the Institute of Pathological Anatomy and Histology of the University of Pisa
}

The statements of many authors on the problem of the penetration of dust particles into the connective tissue of the lung appear to contradict the results obtained from morphological and experimental observations.

Many writers, noting the frequent appearance of dust cells in the lumen of the alveoli and their apparent resemblance to dust-laden cells in the interstitial deposits in pneumoconiosis, accept the view that the endoalveolar phagocytes loaded with inhaled dust actively penetrate the alveolar walls by amoeboid movement, and hence work their way into the connective tissue and into the lymph channels, as far as the storage sites.

The study of the alveolar dust cells has been too closely bound up with histological questions about alveolar lining and with its embryological derivation. It seems unnecessary to decide whether or not a phagocyte is endodermal in origin before admitting that the phagocyte itself acts as an epithelial cell. I think I can leave out this aspect of the discussion, making a distinction between the problem of the origin and the nature of the alveolar phagocytes, and the participation of these cells in the absorption of dust in the connective tissue spaces of the lung.

Many experimental and morphological studies demonstrate that the activity of the dust cells is mainly evident on the surface of the bronchioloalveolar spaces.

Several facts must be borne in mind. The phagocytosis of substances, inhaled or inoculated into the trachea, takes place with a rapidity (about 10 minutes) that demonstrates the superficial position of the phagocytes. This proves that they are not of migratory origin from the connective tissue spaces or from the blood (Arnold, 1880 ; Seemann, 1931 ; Westhues and Westhues, 1925; Huguenin and Delarue, 1929 ; Businco and Giunti, 1930). Even the granulocytes which are especially adapted to migration, take more time to migrate (Huguenin and Delarue, 1929; Aschoff, 1936) when an exudative lesion occurs.

The intracellular deposition of particles on the alveolar walls is of a temporary character. The cells engaged in phagocytosis rapidly become swollen and detached, and are carried away by the mucous flow, both secretory and expulsive, originating from the bronchioles. This process of detachment is partly related to the rate of the intracellular storage of particles, but is favoured by possible inflammatory exudative complications (Huguenin and Delarue, 1929), by alveolar collapse and by the presence of free fluid in it (Westhues and Westhues, 1925). In fact, fixation of the lung extracted from the thorax by intratracheal injection artificially increases the phenomenon of detached dust cells (Macklin, 1950 and 1951).

This process leads chiefly to expulsion via the bronchi. Some of the inhaled particles, however, reach the pulmonary lymph flow. This occurs almost instantaneously. Blood aspiration in the lungs experimentally observed in animals (Nothnagel, 1877 ) or accidentally in man through rupture of an aortic aneurysm into the trachea (personal observation) is accompanied by the presence of red cells in the pulmonary lymph channels. The time in which this process takes place, measured also with special experiments of intratracheal inoculation, is sometimes less than 10 minutes. This time is too short for it to have occurred through a phagocytosis on the alveolar surfaces (Gillilan and Conklin, 1938).

Later, but not before two or three hours after the inhalation or inoculation into the trachea of particulate matter, particles are seen inside the cells in the peribronchiolar or periarterial connective tissue (Huguenin and Delarue, 1929). Some writers believe that these cells have migrated from the alveoli by their own movement or through passive transport in the lymph, although it is difficult to imagine that cells in an epithelial position, 
or free in the alveolar lumen, could penetrate actively into the interstitium.

The phagocytic content gives rise to a change in the shape of the cytoplasm which becomes globular. Although, as far as I am aware, precise studies are lacking on the subject, this change corresponds with a diminution of those qualities of the surface which go to determine the character of amoeboid activity. I agree with Macklin (1950) that the dust cells quickly become spent phagocytes which are incapable of movement and destined to a passive external elimination. It is admissible that some phagocytes from the alveolar lumen may enter the connective tissue. The process may, however, be similar to that observed in the case of some other substances, such as dust particles or red cells, which can definitely be absorbed without migratory phenomena. I have, in exceptional cases, found in the regional lymph nodes of workers who have died from pulmonary asbestosis, some asbestos needles, about the length of 20 cells ; in such cases a passive transport by the lymphatic flow is the only admissible form of transport.

Other writers, while recognizing the impossibility of endocellular transport from the alveolar lumen to the connective tissue, believe that the migration of the phagocytes occurs within the limits of the pulmonary connective tissue spaces, as far as the permanent storage depots. This view corresponds with a current opinion, although positive evidence is entirely lacking. The fact that in the permanent interstitial deposits the dust is incorporated in cells, does not demonstrate that transport has occurred by means of those cells.

However, several observations are in favour of the metastasis of naked particles by means of the lymphatic flow. Akazaki's (1936) experimental findings with the inhalation of soot and powdered quartz should be particularly borne in mind. I, too, have noted in research on human pneumoconiosis that phagocytes in migratory posture or with strangled cytoplasm are never seen in the connective tissues, and that only plump phagocytes are present. I have also noted that, whereas phagocytes are usually not seen on the interalveolar walls, they are present in the permanent interstitial deposit. However, isolated anthracotic particles or naked quartz crystals (recognizable by their double refraction) are often found on the interalveolar walls in patients with silicosis.

Thus there is considerable evidence, although partly negative and indirect, against the hypothesis of endocellular migratory transport of dust. This leads to the admission that transport in the form of naked particles prevails. However, even this concept has not been completely worked out as yet.
A particle of dust, having once reached a lymph channel, may be transported by the lymph flow as far as a lymph node or even into the blood. At lymph node level, it may easily be arrested and hence enter the lymphoreticular tissue of the lymph node itself. At first, it is included in a reticulum cell of the lymph sinus, then this cell sinks into the underlying lymphoreticular tissue, probably not as a result of active migration, but because the other cells on the walls of the sinus (reticulum cells and lymphocytes) grow through an ordinary reactive hyperplastic process. In fact, the lymph node in the course of its life usually grows at the periphery, particularly in the vicinity of the peripheral sinuses, whereas it becomes atrophic centrally and at the hilum. The phagocytes of the sinus, when loaded with particles, take little or no part in the growth, and remain fixed to the old stroma which gradually becomes sclerotic and withdraws, while the active hyperplastic parts advance towards the periphery. Therefore the anthracotic lesion is concentrated far from the sinuses. Analogous phenomena occur in silicosis in the lymph nodes, although disturbed by sclero-hyaline changes (Mottura, 1936, 1938).

A process of this kind seems to occur only in the lymph nodes or in granulation tissue, and is probably impossible on the surface of the pulmonary alveoli, where primary penetration of dust into the interstices occurs without hyperplastia.

The pulmonary alveolus is covered by a superficial fluid veil which, at any rate where the wall is practically free of cells, must be physically continuous with the interstitial fluid. The foreign bodies which escape from the phagocytic barrier remain incorporated in this film, as in a foam bubble, and are possibly carried into the interstitial flow.

Further, it is well known that the lymph channels of the lung originate not in the interalveolar walls, but in the adventitial connective tissue of the bronchioles and the terminal branches of the arterioles (Miller, 1947 ; Luna, 1937 ; Ottaviani, 1938). On the other hand, it is universally admitted that the entry of the particles occurs at the alveolar surface. Thus an interstitial space exists, and extends from this surface as far as the origin of the lymph channels. Here lymph exists in the form of interstitial fluid, not canalized but rather retained according to McMaster and Parsons' (1939) idea of connective tissues in general. Here, as in other connective tissues, it must be admitted that fibrous structures, microscopically visible, such as reticular fibres (Volterra, 1927; McMaster and Parsons, 1939), or even fibrous ultramicroscopic multimolecular structures (Frey-Wyssling, 1948), guide the interstitial fluids. Free fluid reaches the surface only 
as oedema or canalized lymph. The particles necessarily follow this flow before being admitted into the lymph channels. This is the most difficult point to understand, but an explanation in these terms does not seem impossible, whereas the postulate of an active migratory cellular mechanism does not make it clearer.

All connective tissue, faced with foreign matter, such as dusts inoculated by tattooing, red cells, etc., shows an increase in lymphatic drainage, so that, under many conditions, the origin of the lymph channels must be considered as functionally open to the entry of even very bulky impurities. The pulmonary connective tissue, in comparison with that of other organs, is much more easily invaded by external substances, e.g. in the common condition pneumoconiosis caused by soot or quartz.

Phagocytosis in the connective tissue of the lung usually occurs when the particle has already passed the interval corresponding with the interalveolar walls. In experimental pneumoconiosis in animals and in man, all investigators note that the interalveolar walls are almost always free of phagocytes. Thus, it may be affirmed that the incorporation of dust particles in the interstitial cells occurs as a temporary stage or as a permanent deposit in sites different from the interalveolar walls, where entry from outside occurs.

In early experiments on anthracosis from soot (Ruppert, 1878 ; von Ins, 1878) it was noted that the dust-laden cells were found with some regularity where the pulmonary connective tissue was thickest, and especially around the large blood channels. It was thought that this corresponded with particular regions of the lymph apparatus. This hypothesis was forgotten until it reappeared in the works of Gardner, 1933, and of Simson and Strachan, 1931.

Simson and Strachan (1931) made a plastic reconstruction by means of serial sections of pulmonary tissue from a case of status lymphaticus. They found that peripheral accumulations of lymphocytes were situated mainly in three areas : in the connective tissue between the bronchioles and the adjacent branches of the pulmonary artery; at the junction of the small veins of the primary and secondary lobules with the large branches of the pulmonary vein; and along the course of the pulmonary artery and in the connective tissue below the pleura. They thought that in pneumoconiosis these areas were collection sites for the phagocytes migrated from the lymph channels. However, no confirmation of this hypothesis was obtained.

Later, Simson (1935) reconstructed two pieces of lung containing silicotic nodules. He pointed out the relation between the nodule and the branching respiratory bronchioles. (Furthermore, in the photographs of the models, arterial branches can be seen in the centre of the nodules.) $\mathrm{He}$ did not find any branches of the pulmonary veins related to the nodules. The writer abstained from precise references to lymph tissue, and declared that he found great difficulty in identifying this amongst the gross changes due to silicotic sclerosis.

I took up the problem, in collaboration with Dulbecco, by means of reconstruction models of serial sections of non-sclerotic pneumoconiosis, for example, anthracosis and simple siderosis, and of nodular silicosis in early stages, in various cases and with varying magnifications (Mottura and Dulbecco, 1946 and 1947 ; Mottura, 1951).

In anthracosis and siderosis, we found intracellular deposits selectively situated in the adventitial connective tissue of the distal branches of the pulmonary artery and of the corresponding respiratory bronchioles of all sizes. That is to say, these deposits are contained in strands of axial connective tissue of the secondary and primary lobules, while the alveolar connective tissue and, for the most part, the interlobular connective tissue, remain undamaged.

In nodular silicosis of iron moulders the intracellular dust deposits were found not only in the same axial positions in the lobules, but also around the lobules. In silicosis, as is well known, a greater development of the connective tissue occurs in relation to the deposits. The changes, which make the picture difficult to interpret, are derived from this and from the increased emphysema which occurs besides the sclerotic centres (perisclerotic emphysema, Mottura, 1941). Axial sclerosis, particularly in periarterial and peribronchial areas, appears in the form of strands swollen into nodules due to focal hyaline transformation. The silicotic nodules preferentially tend to be situated at the beginning of these axial sclerotic strands, that is, at the junction of the relatively undamaged interalveolar connective tissue with the periarterial and peribronchiolar connective tissue, where, ascending the bronchiol-alveolar tree, the dust deposits begin to appear (Fig. 1).

Interlobular sclerosis, that is sclerosis peripheral to the lobule, appears as extensive and thick sheets of dense connective tissue, which include the vein branches. It is known that these, in fact, usually run between the lobules (Miller, 1947). The larger sclerotic sheets (between the secondary lobules) give off branches which may take the form of cusps, ascend along the vein branches between the primary lobules, and can be followed in serial sections until they join up with the axial sclerosis. These branches accompany the small venous channels, described by Miller (1947), originating 


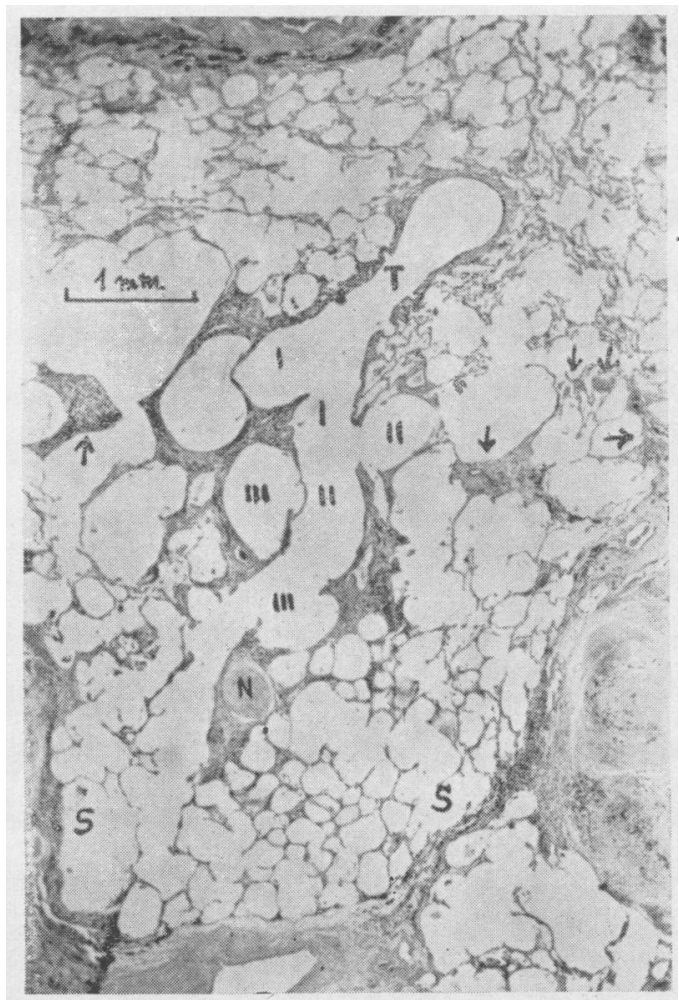

FIG. 1.-Section of a secondary lobule from a silicotic lung (simple nodular silicosis in a moulder).

$T=$ terminal bronchiole.

I, II, III = respiratory (alveolar) bronchioles of first, second, and third order. (They correspond to the axial sclerosis in the lobule.) $\mathbf{N}==$ silicotic nodule at the end of the bronchiolar tree.

$\mathbf{S}=$ interlobular sclerotic septa (peripheral laminar sclerosis in the lobule).

The arrows show sclerotic perivenous strands, which join radially the interlobular sclerosis with the axial one.

from the lobular axis and extend to its periphery, and perhaps correspond with the anastomotic arterio-venous branches which run radially within the lobules. In these sites of laminar interlobular sclerosis, the hyaline changes are mostly diffuse and not nodular, although true nodules are observed, particularly at the apex of the branches directed radially towards the axis of the lobule. Therefore, I distinguish two fundamental topographical components of silicotic sclerosis : axial sclerosis of the secondary and primary lobules, in the form of strands and nodules (periarterial and peribronchiolar); and interlobular sclerotic sheets between the secondary lobules (perivenous), with radial projections between the primary lobules which may join the interlobular sclerosis with the axial sclerosis. Interlobular sclerotic sheets are mainly developed near the pleura. It is clear that all these lesions follow the course of the lymph channels, the latter running chiefly in the adventitia of the axial (arteries) and peripheral (veins) blood vessels of the lobules.

Wulff (1934) had already pointed out in his observations on silicotic lung sections that the interlobular septa suffer severe fibrous changes and may branch out towards the centre of the lobule. This writer interprets silicosis as an obliterating lymphangitis. He affirms that the drainage of the dust occurs at first along the easiest path of the lymph channels following the roots of the pulmonary vein, therefore the silicotic changes would develop in the first instance and most intensely at the periphery of the lobule, and only afterwards, when the peripheral pathways are blocked, would they advance towards the centre of the lobule.

Contrasting with this interpretation is the fact that in pneumoconiosis caused by inert dusts we found the deposits almost exclusively along the axial lymph channels, which would thus appear to be selectively damaged. Also in early silicosis we found great development of axial sclerosis. Therefore it would appear that the interlobular sclerosis is secondary to the blocking of the principal axial drainage channels, and is due to dissemination of dust along the collateral peripheral pathways. The next question is, Do the pneumoconiotic deposits correspond to certain determined tracts of the pulmonary parenchyma?

The elective areas of inert dust deposits and silicotic nodules correspond, broadly speaking, to regions where, in certain rodents and occasionally also in man, lymphoid accumulations are found. This correspondence has been noticed by various writers who think that the dust deposits occur in preconstituted lymphoid stations. Nevertheless, in the great majority of human cases, it would be necessary to consider them not so much as lymphoid stations already existent, but rather as hystiocytic hyperplastic foci in predetermined sites. It is important to understand the limits of this predetermination and also their exact relationship with the lymph channels in general.

Clumps of hyperplastic histiocytes are not found in the lymph channels in pneumoconiosis but in the connective tissue in immediate relationship to them, and more precisely in the connective tissue near their beginning. In connective tissue interstices in general, invasion by foreign bodies may be resolved by simple lymphatic drainage. Only particularly irritating or excessively abundant or stagnating substances provoke cellular reactions, including phagocytosis, which expresses the insufficiency of lymphatic drainage, and takes place before the origin of the lymph channels. I think it probable that every accumulation of dust-laden phagocytes is an expression of the impossibility 
or inadequacy of a prompt drainage of interstitial accumulations. For this reason pneumoconiosis should not at first be considered as a disease of the lymph channels, but as a disease of the interstitial roots of the lymph channels. Naturally diseases of the lymph apparatus, such as involvement at lymph node level, may afterwards aggravate the situation in the peripheral tissues. Therefore, when we note the electivity of the deposits in the peripheral bronchiolo-arterial field in relation to the origin of the principal lymph channels, we may postulate that this is due to the effect of a concentration of particulate substances, which are difficult to drain, rather than a special predisposition or liability to phagocytic activation in the connective tissue in this field.

When, in the phagocytic accumulation, the dust provokes particular progressive changes, such as hyperplasia of cells and fibrils and hyalinosis, then further hindering factors intervene. Thus the dissemination of the same process along collateral pathways, which run within the interlobular septa, is provoked. The development of this process in silicosis causes the silicotic nodules. In the granulomatous and massive forms, the addition of an early diffuse hyperplasia complicates the process and less characteristic topography results.

The silicotic nodule acquires its specific properties chiefly because of its situation, which usually corresponds to a certain distal tract of the arteriolar and broncho-alveolar branches. This is probably not due to a strict specific property of the tissue, but to an accidental though frequent concurrence of circumstances, which determine the stagnation of interstitial lymph flows at a level where convergence and concentration occur.

\section{REFERENCES}

Akazaki, K. (1936). Beitr. path. Anat., 97, 439

Arnold, J. (1880). Virch. Arch. path. Anat., 80315.

Aschoff, L. (1936). Verhandl. deutsch. Gesell. inn. Med., 48, 100.

Businco, A., and Giunti, G. (1930). Haematologica, 11, 499.

Frey-Wyssling, A. (1948). "Submicroscopic morphology of protoplasm and its derivatives," New York-Amsterdam. Trans. by J. J. Hermans and M. Hollander.

Gardner, L. U. (1933). J. Amer. med. Ass., 101, 594 ; (1937), J. ind. Hyg. a. Toxicol., 19, 111.

Gillilan, L. A., and Conklin, R. E. (1938). Amer. J. Physiol., 123, 598 Ins, A. von, (1878). Virch. Arch. path. Anat., 73, 151.

Huguenin, R., and Delarue, J. (1929). Ann. Anat. path., 6, 1181.

Huguenin, R., and Delarue, J. (1929). Ann.
Luna, E. (1937). Riv. Patol. sperim., 8, 70.

Macklin, C. C. (1950). Proc. Inst. Med. Chicago, 18, 78 ; (1951), Lancet, 260, 432 .

McMaster, P. D., and Parsons, R. J. (1939). J. exper. Med., 69, $247,265$.

Miller, W. S. (1947). “The Lung," 2nd ed., Springfield.

Mottura, G. (1936). Arch. it. Anat. e Istol. patol., 7, 449 ; (1938). Giorn. R. Accad. M2d. di Torino, 101, 315; (1941). Assist. soc. 15, n.4; (1951). Med. d. lavoro, 42, 81 .

Mottura, G., and Dulbecco, R. (1946). Rass. Med. industr., 15 82 , (1947) Bull, d'histol. appl., $24,11$.

Nothnagel, H. (1877). Virch. Arch. path. Anat., 71, 414.

Ottaviani, G. (1938). Morph. Jb., 82, 453.
Ruppert, H. (1878). Virch. Arch. path. Anat., 72, 14.

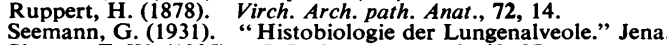

Simson, F. W. (1935). J. Path. a. Bacteriol., 40, 37.

Simson F. W., and Strachan, A. S. (1931). Public. South Afr. Inst Med. Res., No. XXVI 2, 6, vol. 4, p. 231.

Volterra, M. (1927). Sperimentale, 81, 319.

Westhues, H., and M. (1925). Beitr. path. Anat., 74, 432.

Wulf, H. B. (1934). Acta path. microb. Scand., 11, 389. 